\title{
Specific solution of problem of water filtering in the rocks by the finite element method
}

\author{
Olena Slashchova ${ }^{1, *}$, Ihor Slashchov ${ }^{1}$, and Iryna Sapunova ${ }^{1}$ \\ ${ }^{1}$ Institute of Geotechnical Mechanics named by N. Poljakov of National Academy of Sciences of \\ Ukraine, 49005, Dnipro, Simferopolska Str., 2a, Ukraine
}

\begin{abstract}
The article is devoted to development of methods for geofiltration calculations with taking into account peculiarities of changes of the rock physical and mechanical properties at water saturation. Methods: mathematical modeling of geomechanical and filtration processes with the help of finite element method and laboratory and underground studies. A mathematical model was formulated for solving a problem of elasticity theory by the finite element method, which took into account peculiarities of water-saturated rocks. Pattern of stress-strain state changing in the fractured water-saturated rocks under the action of critical loads, which occurred around the preparatory roadways during their operation, were established. In order to solve the filtration problems, a bank of collected initial data on physical and mechanical properties of water-saturated rocks was processed with the help of variation coefficients, which were taken into account by the method, which assumed calculation of the model loading with critical parameters.
\end{abstract}

\section{Introduction}

Underground mining of minerals almost always disturbs aquifers. However, the undermined aquifers change groundwater behavior, direction and intensity of water stream flowing into the fracture systems and, as a result, saturates the rocks, hence, essentially degrading their strength. Such hydro-geomechanical processes lead to intensive uncontrolled deformation of preparatory roadways, which, in their turn, become additional contours of the aquifer relieve. Therefore, it is obvious that study of the processes that occur during the mining activity require combined solution of geomechanical and filtration problems [1-5]. At the same time, a reliable prediction of only geomechanical state of the rock massif is complicated by the use of idealized models and generalized empirical dependencies, which do not take into account a number of factors specific for real objects under the study $[1,4]$. It is also quite difficult to create a filtration model adequate to real conditions due to the uncertainty of changes of the following basic filtration parameters: permeability coefficients, boundary conditions for specifying conditions for aquifer in terms of area and time, and anisotropy of water-bearing rocks.

Practice shows that researches intended for reducing water inflows into the roadways

\footnotetext{
*Corresponding author: OASlashchova@nas.gov.ua
} 
under the influence of mining activity have not bring satisfactory results on preventing roof rock fall and floor heaving because it is rather difficult to determine in advance rate of water-saturated rock influence on stability of concrete roadway due to a number of objective reasons. First, despite availability of serious studies on determining stress-strain state of the rock massif, there are no reliable mathematical models, which take into account specificity of filtration processes occurred in water-saturated rocks. Secondly, initial parameters of the rock physical and mechanical properties can be subjective and do not reflect the real object, hence, leading to miscalculation. Thirdly, patterns of the stress-strain state changes in the fractured water-saturated rocks under the effect of critical loads, which occur around the preparatory roadways during their operation, are not well studied. Therefore, scientific task aimed at developing methods for geofiltration calculations with considering specific changes of physical and mechanical properties of water-saturated rocks, is a pressing challenge for today.

\section{Methods}

Research methods: analysis and generalization of literature and experimental data; mathematical modeling of geomechanical and filtration processes by the finite element method; underground studies of the rock physical properties by standard methods and equipment; statistical processing of measurement results.

\section{Results and discussion}

In order to solve filtration problems, as well as when solving elasticity theory problems, area under the study is divided into elements with finite sizes, preference is given to triangular elements. Within each element, pressure gradients $d H / d x$ and $d H / d y$ are assumed to be constant, therefore, the pressure function within one element can be expressed as a linear function of Cartesian coordinates [6]:

$$
H=a_{1}+a_{2} x+a_{3} y,
$$

where $a_{1}, a_{2}, a_{3}-$ are linearization constants. In the matrix form, it is:

$$
\{H\}=\left\{\begin{array}{l}
H_{i} \\
H_{j} \\
H_{k}
\end{array}\right\}=[A]\{a\}=\left[\begin{array}{ccc}
1 & x_{i} & y_{i} \\
1 & x_{j} & y_{j} \\
1 & x_{k} & y_{k}
\end{array}\right]\left\{\begin{array}{l}
a_{i} \\
a_{j} \\
a_{k}
\end{array}\right\},
$$

where $\{H\}$ is pressures vector; $[A]$ is coordinate matrix, $\{a\}$ is vector of parameters. By differentiating equation (1), we obtain the expression for the pressure gradients:

$$
d H / d x=a_{2} ; \quad d H / d y=a_{3} .
$$

Let's write (1) in the matrix form:

$$
\{d H\}=[B]\{a\}
$$

where $B=\left[\begin{array}{l}010 \\ 001\end{array}\right]$ is matrix of coupling.

From equation (2), we have: 


$$
\{a\}=[A]^{-1}\{H\} .
$$

By substituting it into (4), we get

$$
\{\partial H\}=[B][A]^{-1}\{H\} .
$$

Filtration process follows the Darcy law:

$$
\left\{k_{f}^{\prime}\right\}=\left[k_{f}\right]\left\{d H^{\prime}\right\}
$$

where $\left\{v_{f}^{\prime}\right\}=\left\{\begin{array}{l}v_{x}^{\prime} \\ v_{y}^{\prime}\end{array}\right\}$ and $\{d H\}=\left\{\begin{array}{l}d H / d x^{\prime} \\ d H / d y^{\prime}\end{array}\right\}$ are velocity vectors and, accordingly, pressure gradients along the axes $x^{\prime}, y^{\prime} ;\left[k_{f}\right]=\left[\begin{array}{cc}k_{x}^{\prime} & 0 \\ 0 & k_{y}^{\prime}\end{array}\right]$ is matrix of filtration properties; $k_{x}^{\prime}, k_{y}^{\prime}$ - are filtration coefficients along main directions.

$$
\left\{v_{f}^{\prime}\right\}=[C]\left\{v_{f}\right\} ; \quad\left\{d H^{\prime}\right\}=[C]\{d H\},
$$

$$
\begin{aligned}
& \text { where }[C]=\left[\begin{array}{rr}
\cos \alpha & -\sin \alpha \\
\sin \alpha & \cos \alpha
\end{array}\right] \text { is indicative matrix. } \\
& \qquad\left\{v_{f}\right\}=\left[k_{f}\right][C][C]^{-1}[B][A]^{-1}[H],
\end{aligned}
$$

where $\left[k_{f}\right][C][C]^{-1}$ is equal to $k_{f}$ for isotropic medium. By analogy with elasticity theory, and considering that additional work of external forces is equal to additional work inside the contour, one connects additional pressure with additional water inflows through the matrix, which is similar to the system stiffness matrix.

Thus, in isotropic media, formulation of solution of the plane problem of elasticity theory solved by the finite element method and the program for its implementation can, with an insignificant correction, be used for solving plane filtering problems (and similar problems). The essence of this correction lies in the fact that Poisson ratio $\mu$ and elastic modulus $E(\mathrm{MPa})$ are changed in elastic matrix $[D]$, which, for the case of plane deformation, has the form [6]

$$
[D]=\frac{E}{(1-2 \mu)(1+\mu)}\left[\begin{array}{ccc}
1-\mu & \nu & 0 \\
\nu & 1-\mu & 0 \\
0 & 0 & 1-2 \mu / 2
\end{array}\right],
$$

the Poisson ratio is assumed to be 0.5 (for incompressible medium), and element $\varepsilon_{33}$ of the deformation matrix is multiplied by two (in this case, form of the matrix does not matter). Further, let's assume that all nodes of the area feature zero movement along the $y$ axis, and let's apply filtration coefficient $k_{f}$ (or thermal conductivity in thermal problems) instead of elastic modulus $E$ and nodal inflows and pressure instead of the given nodal forces and displacements, respectively. Then, as a result of solving the problem, we obtain nodal pressures instead of nodal displacements along the axis $x$, pressure gradients $d H / d x$ instead of deformations $\varepsilon_{x}$, pressure gradients $d H / d y$ instead of deformations $\gamma_{x y}$, filtration rate $v_{x}$ 
instead of stress $\sigma_{x}$, and filtration rate $v_{y}$ instead of stress $\tau_{x y}$.

Preparation of initial information is a rather laborious process as each specific geomechanical and filtration problem requires construction of a new scheme (model) [7]. Furthermore, elements of subjectivity and randomness are typical phenomena in mathematical model at its dividing into elements; initial parameters of the rock strength, as a rule, do not reflect a real object, and often are absent at all, hence, affecting final results of calculations and their reliability. Geological services do not always have an opportunity to take samples for testing or they take them in minimum amount, though variation of parameters of the rock physical and mechanical properties can reach up to $30 \%$ within one mine roadway. Effect of fractured rocks is either not taken into account at all, since rocks, when they are sampled, split along the cracks, or are taken into account indirectly. Therefore, in order to calculate parameters of the underground roadway stability, it is proposed to use averaged indicators of the rock physical and mechanical properties with taking into consideration depth and conditions of occurrence, stage of katagenesis, and, which is the most important, coefficients of parameter variation. The coefficients of variation are taken into account by the method, which assumes calculating the model at critical (minimum possible) and averaged strength parameters. If necessary, with the view to higher reliability of calculation results, it is possible to bring to compliance with specific mining and geological conditions basing on testing a minimum number of samples or conducting a limited amount of geophysical measurements, though, without relying on high representativeness of the samples taken.

The Institute of Geotechnical Mechanics named by N. Poljakov of National Academy of Sciences of Ukraine obtained a great number of factual data as a result of research of the rock physical and mechanical properties. In Table 1, averaged basic parameters are shown, which characterize deformation and strength properties of the rocks: $E$ is elastic modulus, $\mu$ is Poisson ratio, $\gamma$ is bulk density, $C$ is adhesion, $\varphi$ is angle of internal friction, $\sigma_{e}$ and $\sigma_{0}$ are limits of compressive and tensile strength, respectively.

Table 1. Averaged data on physical and mechanical properties of rocks.

\begin{tabular}{|c|c|c|c|c|c|c|c|}
\hline Rocks & $\begin{array}{c}E \cdot 10^{-4} \\
\mathrm{MPa}\end{array}$ & $\mu$ & $\begin{array}{r}\gamma, \\
\mathrm{t} / \mathrm{m}^{3} \\
\end{array}$ & $\begin{array}{c}C, \\
\mathrm{MPa} \\
\end{array}$ & $\begin{array}{c}\varphi, \\
\text { grade }\end{array}$ & $\begin{array}{c}\sigma_{e}, \\
\mathrm{MPa}\end{array}$ & $\begin{array}{c}\sigma_{0}, \\
\mathrm{MPa}\end{array}$ \\
\hline Sandstone $^{1}$ & 3.52 & - & - & - & - & 147 & 2.7 \\
\hline Sandstone $^{2}$ & $2.0-4.0$ & $0.1-0.3$ & 1.9 & 15.0 & 38 & $72-100$ & - \\
\hline Sandstone $^{3}$ & 4.47 & 0.28 & 2.55 & 27.0 & 45 & 74 & 9 \\
\hline Sandstone ${ }^{4}$ & $1.8-2.2$ & $0.24-0.25$ & $2.4-2.6$ & 20 & $30-32$ & - & - \\
\hline Siltstone $^{2}$ & $1.0-3.0$ & $0.2-0.3$ & 2.6 & & 30 & $40-100$ & - \\
\hline Siltstone ${ }^{3}$ & 4.42 & 0.3 & 2.0 & 18.7 & 33 & 52 & 7.9 \\
\hline Siltstone $^{4}$ & 0.5 & 0.15 & - & 20 & 25 & - & - \\
\hline Mudstone $^{2}$ & $1.0-4.5$ & $0.17-0.25$ & - & - & 30 & $38-110$ & - \\
\hline Mudstone $^{3}$ & 3.5 & 0.29 & 2.7 & 13.6 & 41 & 26 & 6.6 \\
\hline Mudstone $^{4}$ & 1.3 & 0.33 & 2.4 & 4 & 20 & - & - \\
\hline Limestone $^{2}$ & $4.0-8.0$ & 0.27 & - & - & 27 & $12-25$ & - \\
\hline Limestone $^{3}$ & 5.0 & 0.2 & 2.71 & - & - & 84 & 10 \\
\hline $\begin{array}{l}\text { Fractured } \\
\text { limestone }\end{array}$ & 10 & 02 & 21 & 3 & 25 & & \\
\hline & 1.0 & 0.2 & 2.1 & 3 & $2 J$ & - & - \\
\hline
\end{tabular}

According to data: ${ }^{1}$ Usachenko B.M .; ${ }^{2}$ Baklashova I.V.; ${ }^{3}$ Grebenkina S.S.; ${ }^{4}$ Kirnichansky G.T.

In order to determine input data for mathematical modeling, dependences of change of sedimentary rock strength under the influence of pressure and water saturation were established. Laboratory studies of more than three thousand samples were systematized. It was determined that rock water saturation led to decrease of their strength by 1.5-2.0 times (for sandstones and limestones) and 2.5-3.0 (for siltstones and mudstones) (Fig. 1).

It was established that rock water saturation during 20 days led to disintegration and 
complete loss of compression resistance in $89.6 \%$ of mudstone samples, $62.0 \%$ of siltstone samples, $3.6 \%$ of limestone samples, $12.8 \%$ of sandstone samples. Tests of mudstones at dry and water-saturated condition showed that increasing of water content in the sample by $2.5 \%$ reduced its compressive resistance by half, and at water content of $5 \%$, many samples lost their ability to resist compression during the first 20 days. During the same period, more than half of siltstone and limestone are soaked and completely lost their ability to resist compression. Sandstones, in most cases, were weakened slightly, and period of their disintegration, more often than not, exceeded 20 days.

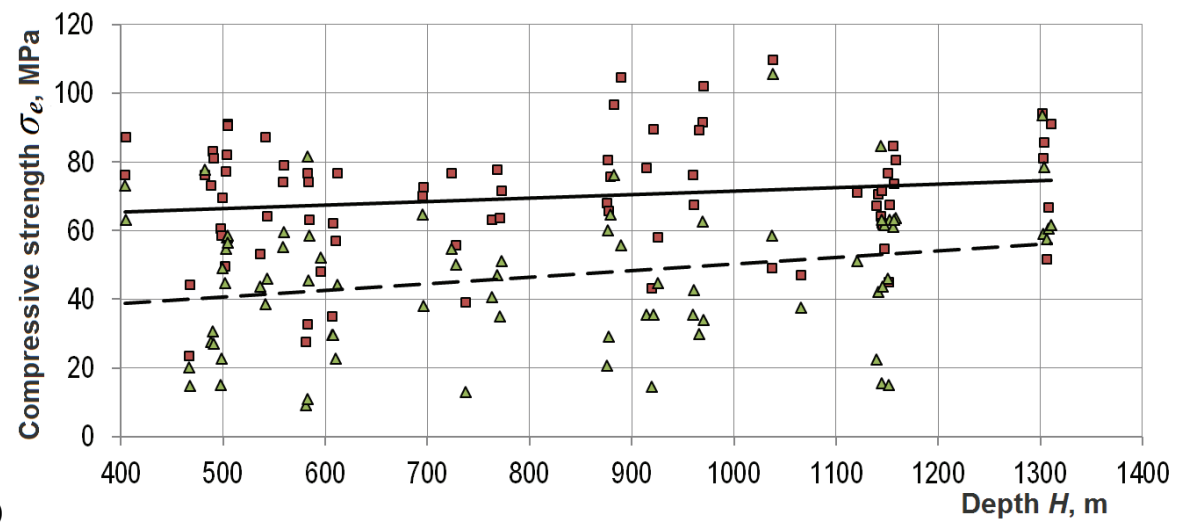

a)
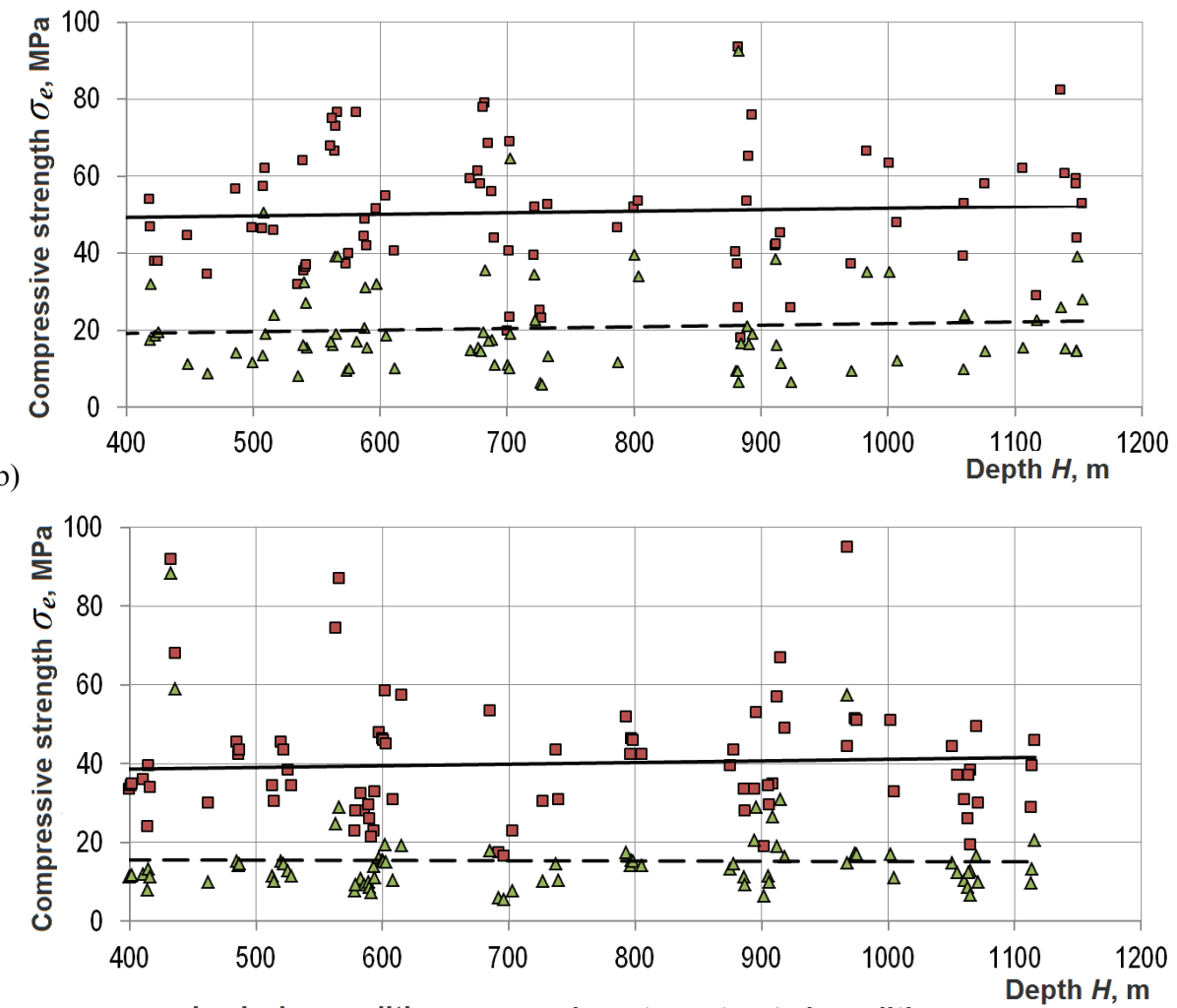

c)

Fig. 1. Patterns of reducing the compressive strength of sandstones (a), siltstone (b) and mudstone (c) at water saturation with consideration of residual strength of completely disintegrated rocks. 
A similar result is shown by B.M. Usachenko on determining speed and shape of the rock disintegration in water. Ninety eight percent of all tested mudstone samples, $88 \%$ of siltstones, and $26 \%$ of sandstone samples related to canal coals are completely disintegrated and transformed into a shapeless mass. At the same time, rocks related to gas coals are more resistant to disintegration: $69 \%$ of siltstone samples and $87 \%$ mudstone samples were disintegrated. Sandstones, in most cases, are weakly disintegrated in water. Intensity of clay rock deformation depends on their mineralogical composition. It should be noted that strength of sedimentary rocks decreases at water saturation and is linearly dependent on the initial strength. At the same time, the clay rocks are soaked completely, therefore, in the computational methods, their residual strength can be taken as strength of completely disintegrated rocks (Fig. 1).

Increase of clay fraction content contributes not only to strength decrease at water saturation, but also to greater variation of strength parameters (i.e. increase of variation coefficient). The coefficient of variation was determined from the ratio

$$
c_{V}=\sqrt{\frac{1}{n} \sum_{1}^{n}\left(x_{i}-\bar{x}\right)^{2}} / \bar{x},
$$

where $x_{i}$ is $i$-th element of the sample; $n$ is the sample; $\bar{x}$ is sample average.

It is established that coefficients of rock strength variation depend on the rock water saturation (Fig. 2). At water saturation, rocks have a greater variety of average values of the variation coefficients than in dry state. For mudstones and siltstones, change of this coefficient is approximately $48-58 \%$ and for sandstones and limestones is about $44-52 \%$. That is, an increase of rock strength up to $60 \%$ is observed at water saturation in comparison with variation of rock strength in the dry state. Values of variation coefficients allowed carrying out calculations with critical parameters of rock strength (minimum strength) both in dry and water-saturated state.

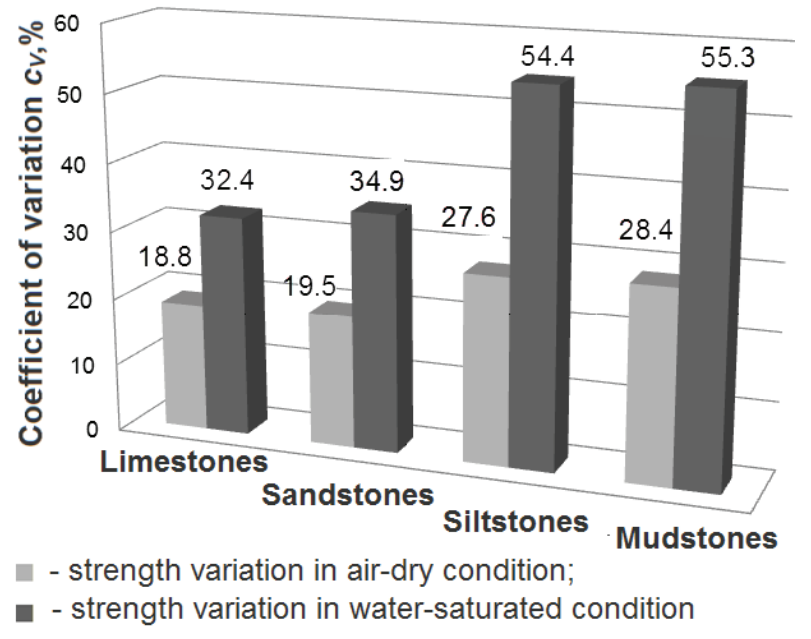

Fig. 2. Changes of variation of rock compressive strength depending on the rock water saturation with taking into account residual strength of completely disintegrated rocks.

The method of geomechanical calculations based on parameter average values with taking into account variation coefficients was used by the author of [8] in order to develop an information system for the safety underground mining activity.

According to the proposed method, initial data for modeling stress-strain state of the 
rock mass are averaged indicators of the rock physical and mechanical properties. Under consideration was underground roadway located in a transversely isotropic medium at the depth of $1280 \mathrm{~m}$. Imitation of sequential watering of rocks in the underground roadway was achieved by a gradual decrease of strength properties of the model elements, which corresponded to the disintegrated and water-saturated areas of the rock massif. Filtration features of rocks and open porosity coefficients were taken into account. Values of acting principal stresses were determined (Fig. 3).

Investigated the stress-strain state of the soil during pre-hardening of rocks, which, if applied before the start of watering, significantly changes the nature of the deformations. The unloading zone is replaced by a zone of increased stresses at the hardening sites and indicates an increase in the carrying capacity of the array and a decrease in its filtration properties. Deformations are distributed more evenly across the width of the excavation. Application in the studied conditions of pre-hardening of the soil generation reduces the relative displacement by $18-42 \%$, depending on the volume of water input and the depth of the hardened zone.

It is established that the stress-strain state of the rock massif below the level of the soil generation is unevenly distributed and is characterized by unloading zones in the middle and stress concentration from the sides. Extremely strained soil rocks with increasing water saturation quickly lose their bearing capacity in layers.

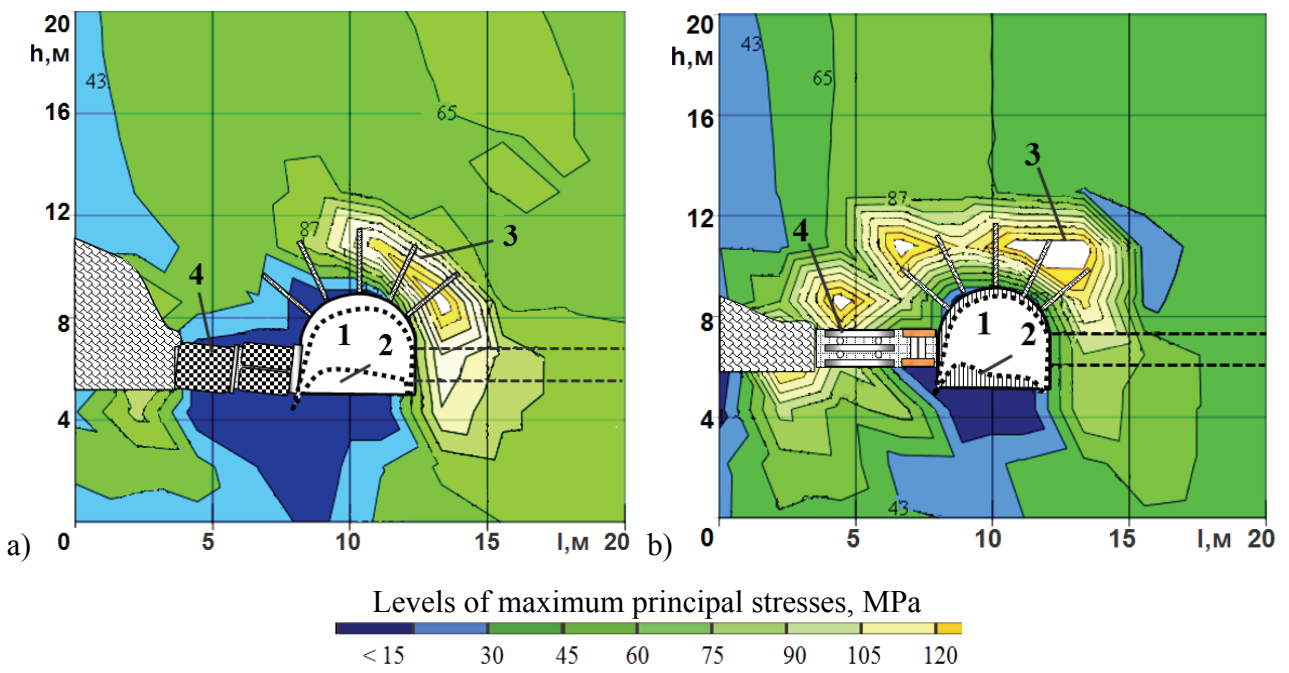

Fig. 3. The calculated maximum principal stresses around the preparatory roadway with bolt-and-arch support using yielding pack (a) and rigid rib-side pack (b): 1 - preparatory roadway; 2 - deformations of roadway floor after water saturation; 3 - roof bolting; 4 - auxiliary measures to protect the roadway.

It is established that stress-strain state of the rock massif below the level of the roadway floor is unevenly distributed and is characterized by relieve zones in the center and by stress concentration in the sides. At increasing water saturation, extremely stressed floor rocks quickly, layer-by-layer, lose their bearing capacity.

The floor stress-strain state is investigated during preliminary rock consolidation, which, if it is applied before the watering starts, significantly changes nature of deformations. Relieve zone is changed into zone of increased stresses at areas of consolidation, being the witness of the fact that carrying capacity of the massif is increased while its filtration properties are degraded. Deformations are distributed more evenly across the width of the roadway. Application of preliminary floor consolidation under the studied conditions reduces relative displacements by $20-40 \%$ depending on the volume of water 
input and depth of the consolidated zone.

Underground studies were conducted in the field gas gathering roadway of the seam $\mathrm{m}_{3}$ in the A. Zasyadko Mine at the depth of $1280 \mathrm{~m}$. Displacements of the roadway floor in the dry (station 23) and water-saturated (station 36) states were measured. Observations show that intensity of heaving is increased significantly due to the floor rock watering during the drilling operations (Fig. 4, Diagram 1). The studies were conducted in similar mining and geological conditions, so the conclusion that difference between the two displacement diagrams is closely connected with the roadway watering during the technological processes (in our case, flushing of the drilling wells with water) is obvious. This fact necessitates consideration of hydrogeological factors when planning technological operations in preparatory roadways.
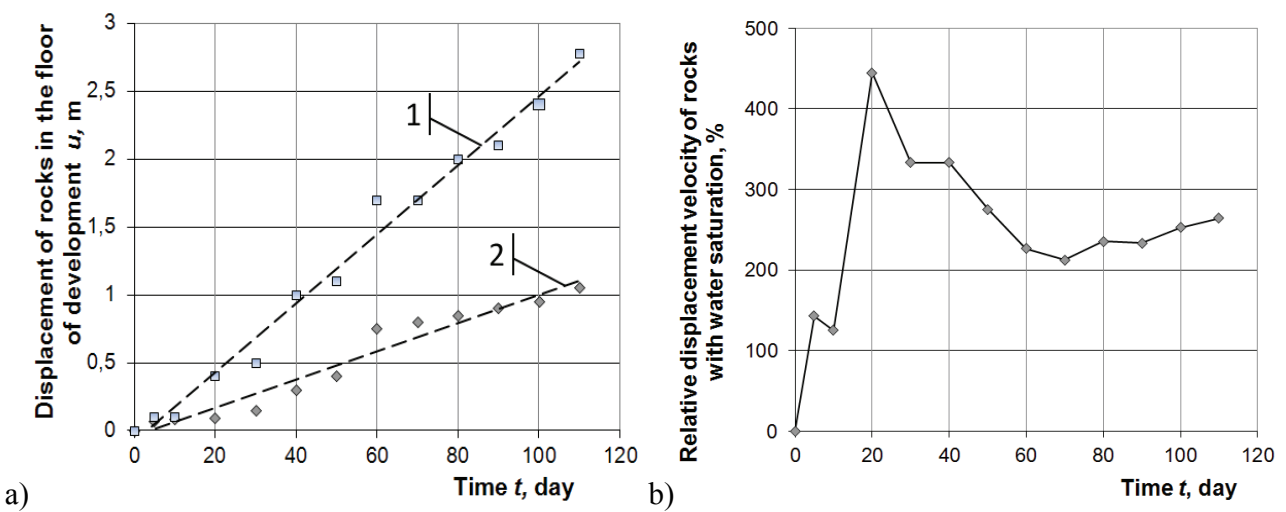

Fig. 4. Dependences between the changes in floor rock displacements (a) and relative rate of heaving change (b) and time period obtained by underground experiments in the field gas gathering roadway of the seam $\mathrm{m}_{3}$, horizon of $1280 \mathrm{~m}$ : 1 - at water saturation (measuring station \#23); 2 - at dry condition (measuring station \# 36).

Processing of results of instrumental measurements at the stations and comparing the data obtained at watering and without watering shows that floor displacements increase over time by linear dependencies and feature close correlation (linear models with water saturation $u=0.026 t-0.08$, and without water saturation $u=0.01 t-0.04, R^{2}=0.95$, Fig. 4, a). Water saturation of the floor rocks demonstrates its maximum effect on stability of the roadway floor during the first 20 days (Fig. 4, b); this fact is confirmed by data of laboratory experiments on the time characteristics of reduced strength of watered rocks. Ratio of heaving rate when water gets the roadway floor to the displacement of the dry preparatory roadway increases 1.5-4.5 times during the first 20 days. Then decrease relative rate of heaving change was observed, and after 60 days of observation, the intensity was stabilized.

\section{Conclusions}

On the basis of the research findings, the following scientific results are obtained:

- a mathematical model was formulated for solving problems of elasticity theory by the finite element method, which differs by taking into consideration deformation of watersaturated rocks. In order to estimate the rock massif stress-strain state, it is proposed to use average values of bank of collected initial data on the rock physical and mechanical properties with variation coefficients, which are taken into account by the method, which assumes calculation of the model loading with critical parameters. In order to improve reliability of results of calculations, it is necessary to take into account concrete mining and 
geological conditions through testing minimum number of samples or conducting limited amount of geophysical measurements;

- analytical methods establish and underground experiments confirm patterns of the stress-strain state changes in the fractured water-saturated rocks under the effect of critical loads, which occur around the preparatory roadways during their operation. Water saturation of the floor rocks demonstrates its maximum effect on stability of the roadway floor during the first 20 days. Ratio of heaving rate, when water gets the roadway floor, to the displacement of dry preparatory roadway increases 1.5-4.5 times during the first 20 days. Then decrease of heaving intensity was observed, and after 60 days of observation, the intensity was stabilized;

- application of preliminary floor consolidation under the studied conditions reduces relative displacements by 20-40\% depending on the volume of water input and depth of the consolidated zone. Therefore, the following actions should be undertaken: to regulate water inflow into the floor during technological operations; to make operational assessment of water effect on the rock strength properties adapted to specific mining and geological conditions and technology; to develop parameters for methods of the floor consolidation with taking into account water saturation.

\section{References}

1. Ikonnikov, M.Yu., Ikonnikov, Yu.R., Slashchova, O.A., Slashchov, I.M., Yalanskiy, A.O. (2015), Matematicheskoye modelirovaniye $v$ zadachakh otsenki effektivnosti $i$ bezopasnosti gornykh rabot [Mathematical modeling in solving problems of evaluating the efficacy and safety of mining operations], NGU MES of Ukraine and IGTM NAS of Ukraine, Dnipropetrovsk

2. Cuss, R.J., Rutter, E.H., Holloway, R.F. (2003), "The application of critical state soil mechanics to the mechanical behaviour of porous sandstones", International Journal of Rock Mechanics \& Mining Sciences, 40, 847-862

3. Szlazak, J., Szlazak, N. (2004), "Numerical determination of methane concentration in goaf space", Committee of Mining and Strata Mechanics Research Institute, 40, 587599

4. Bulat, A.F., Slashchov, I.M., Slashchova, O.A. (2017), "Evaluation methods of interconnected geomechanical and gas dynamic processes in the rock massif for the systems of working medium control in the mines", Geotekhnicheskaya Mekhanika [GeoTechnical Mechanics], 134, 3-21

5. Skipochka, S., Krukovskaya V., Grebenyuk S. (2011), "Investigation of the Deepened Concrete Constructions State in the Process of Operation of Mine Water Spillway", 22nd World Mining Congress, Ankara, 2011, 367-377.

6. Gallager, R. (1984), Metod konechnykh elementov. Osnovy [Finite Element Analysis. Fundamentals], Moskva: Nauka

7. Usachenko, B.M., Slashchova, O.A. (1997), "Study and express-estimation filtrational processes in fissured massifs", Geotekhnicheskaya Mekhanika [GeoTechnical Mechanics], 134, 3-21

4. Bulat, A.F., Slashchov, I.M., Slashchova, O.A. (2017), "Evaluation methods of interconnected geomechanical and gas dynamic processes in the rock massif for the systems of working medium control in the mines", Geotekhnicheskaya Mekhanika [GeoTechnical Mechanics], 59, 34-44

8. Slashchov, A.I. (2016), "Justification of the parameters of the information system assuring the underground mining safety", Naukovyi Visnyk Natsionalnoho Hirnychoho Universytetu, 1, 77-85 